\title{
Haptic terrain classification for legged robots
}

\section{Conference Paper}

Author(s):

Hoepflinger, Mark A.; Remy, C. David; Hutter, Marco (i); Spinello, Luciano; Siegwart, Roland

Publication date:

2010

Permanent link:

https://doi.org/10.3929/ethz-a-010027702

Rights / license:

In Copyright - Non-Commercial Use Permitted

Originally published in:

https://doi.org/10.1109/ROBOT.2010.5509309 


\title{
Haptic Terrain Classification for Legged Robots
}

\author{
Mark A. Hoepflinger, C. David Remy, Marco Hutter, Luciano Spinello, and Roland Siegwart, Fellow, IEEE
}

\begin{abstract}
In this paper, we are presenting a method to estimate terrain properties (such as small-scale geometry or surface friction) to improve the assessment of stability and the guiding of foot placement of legged robots in rough terrain. Haptic feedback, expressed through joint motor currents and ground contact force measurements that arises when prescribing a predefined motion was collected for a variety of ground samples (four different shapes and four different surface properties). Features were extracted from this data and used for training and classification by a multiclass AdaBoost machine learning algorithm. In a single leg testbed, the algorithm could correctly classify about $94 \%$ of the terrain shapes, and about $73 \%$ of the surface samples.
\end{abstract}

\section{INTRODUCTION}

The main advantage of legged locomotion is the ability to move in rough and highly unstructured terrain. In contrast to a wheeled robot, whose motion is limited by the worst obstacle along the continuous path of its wheels, the mobility of a legged robot is only limited by the availability of discontinuous footholds that can be distributed relatively sparse throughout the environment. This allows legged systems to overcome obstacles like grooves, gaps, steps, or sandy and slippery terrain, which might be impassable for vehicles relying on wheels or tracks. However, this ability comes with the drawback that legged robots, due to their intermitted support and the high position of the center of mass, tend to fall and tipp over more easily than wheeled systems. This makes stability a key element when evaluating the performance of legged robots.

Common stability criteria for legged robots can be classified as either static or dynamic. Static methods, such as the center of mass projection method (CM, [1]), completely ignore the motion of the robot and assess the stability solely based on the relative position of the CoM and the feet. This assumption is only valid as long as the robot moves slowly and does not undergo large accelerations. Dynamic stability criteria, such as the Center of Pressure Method (CoP, [2]), or the Zero Moment Point (ZMP, [3]) additionally take into account the current velocity and acceleration of the

Manuscript received September 15, 2009. This work was supported in part by the Swiss National Science Foundation (SNF) (project 200021_119965/1)

Mark A. Hoepflinger, C. David Remy, Marco Hutter and Roland Siegwart are with the Autonomous Systems Lab, Institute for Robotics and Intelligent Systems, Swiss Federal Institute of Technology (ETHZ), 8092 Zurich, Switzerland (+41 4463221 45; fax: +41 44 63211 81; e-mail: markhodethz.ch, cremydethz.ch, mahutterdethz.ch, rsiegwarteethz.ch), Luciano Spinello is with the Social Robotics Lab, Department of Computer Science, University of Freiburg, 79110 Freiburg, Germany; e-mail: spinellodinformatik.uni-freiburg.de

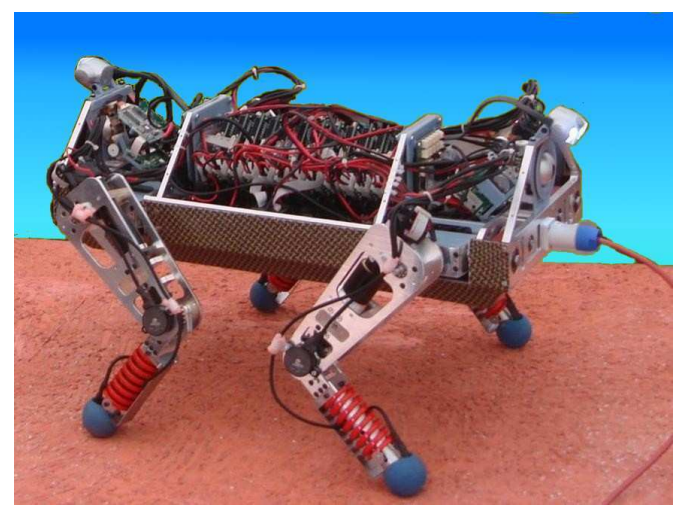

Fig. 1. The presented methodology was developed for the walking robot ALoF, which was specifically designed for locomotion in rough terrain. The robot is about $0.5 \mathrm{~m}$ long and weights about $15 \mathrm{~kg}$.

robot, and can hence not only provide information on footplacement and CoM kinematics, but also give a limit for possible joint accelerations and contact forces. An overview of such stability margins can be found in a comparative study by Gonzalez et al. [4].

However, most of these criteria are solely based on geometric properties and internal states, such as the shape and position of the support polygon in relation to a resulting force on the CoM. This allows simplifying assumptions about the ground, which is consequently often considered to be completely flat, or -in the more sophisticated modelspiecewise planar. Terrain properties in a broader sense, such as friction coefficient, compliance, or small-scale geometry of the ground are not taken into consideration. Yet, these properties will have a very big influence on the actual stability of a walking robot, as they determine the risk of slipping and sliding, and the overall reliability of a stance. Both, under static and dynamic considerations, estimating the terrain characteristic is hence very important for robust and efficient legged locomotion.

Detailed knowledge about the terrain, especially on a small scale, will help to guide foot placement, constrain the CoM motion, or estimate the load-bearing capabilities of a single contact point. In the DARPA learning locomotion challenge [5], [6], for example, detailed geometric terrain information was provided in advance. This information reduced locomotion to a global optimization problem with nearly no uncertainty. As such knowledge is currently not available in real world applications, new ways must be established to characterize an environment while walking through it. In addition to visual perception, physical interaction with the 
TABLE I

DIMENSIONS OF THE ROBOTIC LEG

\begin{tabular}{ll}
\hline Shank length [mm] & 165 \\
Thigh length [mm] & 150 \\
Shank diameter [mm] & 20 \\
Foot diameter [mm] & 44 \\
Position of FSR above the foot [mm] & 90 \\
Leg weight [kg] & 0.64 \\
\hline
\end{tabular}

environment is thereby indispensable. This can be observed in nature, where humans and animals not only visually but also haptically explore possible footholds when it comes to unknown and uncertain terrain. While this issue of terrain classification has drawn considerable attention for locomotion with wheeled [7]-[10] or tracked systems [11], to the best of our knowledge, these principles have never been applied to terrain classification for legged robots.

In this paper, we have applied machine-learning strategies to classify the geometric shape and surface properties of selected footholds based on haptic information. The stance of a robotic leg is investigated in an experimental setup, in which ground elements with different shapes and surfaces were mounted. Small-scale motion is induced, and features are extracted from the resulting contact force and current measurements, and processed by a multiclass AdaBoost classifier [14], [15] to obtain the terrain-sample category. The following section describes the hardware components, the experimental setup, the sampling procedure, and the classification algorithm that were used in this study.

\section{Methods}

\section{A. Robotic leg}

The leg used in our test setup originates from a quadrupedal robot (Fig. 1) designed for research on locomotion in rough and highly unstructured terrain. A single leg (Tab. I) was separated from the main body of the robot and attached to a testbed that could be equipped with different terrain samples. The hip segment was rigidly connected to the sample-holder, which limited the motion of the leg to two degrees of freedom (hip and knee flexion/extension). Only the knee joint was actively driven by an electric DC-motor. The motor and gearbox of the joint for hip flexion/extension were removed in this setup, such that the leg was allowed to swing freely and without drag about its hip axis. Since the rotational axes of the two joints are parallel, the motion of the foot was planar. A compliant spherical rubber ball with a high coefficient of friction was used as foot-element.

\section{B. Force sensing element}

To allow the registration of ground contact forces, the rigid shank of the robotic leg was divided into two parts. The force sensing unit (Fig. 2) was placed at the cutting site which allowed measuring the force along the shank axis as well as the two bending moments in the cutting plane.

In this unit, three individual sensors were uniformly distributed around the leg axis, each one registering the
TABLE II

PERFORMANCE CHARACTERISTICS OF A FORCE SENSING RESISTOR

(FSR-151)

\begin{tabular}{ll} 
Parameter & Value \\
\hline Type: & Interlink Electronics FSR-400 \\
Force Sensitivity Range: & approx. $1 \mathrm{~N}$ to $100 \mathrm{~N}$ \\
Force Repeatability: & $\pm 2 \%$ to $\pm 5 \%$ \\
Force Resolution: & Better than $0.5 \%$ full scale \\
Switch Characteristics: & Essentially zero travel \\
Device Rise Time: & $1-2 \mathrm{~ms}$ \\
Lifetime: & $>10$ million actuations
\end{tabular}

normal force at its position. The upper and lower parts of the sensor were connected with a fixed bolt that exhibited a well-defined pre-compression force onto the sensor layer and additionally enabled the measurement of tensional forces. As sensing elements we used force sensing resistors (Interlink Electronics FSR-400), whose resistance decreases approximately exponentially with the applied force. The most essential characteristics are listed in Table II. We decided to use these sensors, as they are very inexpensive (less than $10 \$$ ) and easy to use, however, we acknowledge that they come with some considerable drawbacks: particularly, they are not suitable for high precision measurements and show a substantial hysteresis which makes them ill suited for highly dynamic measurements. These drawbacks clearly reduce the applicability, but when using them in the field of walking robots and limiting them to tasks such as obstacle detection or terrain classification, their performance is sufficient.

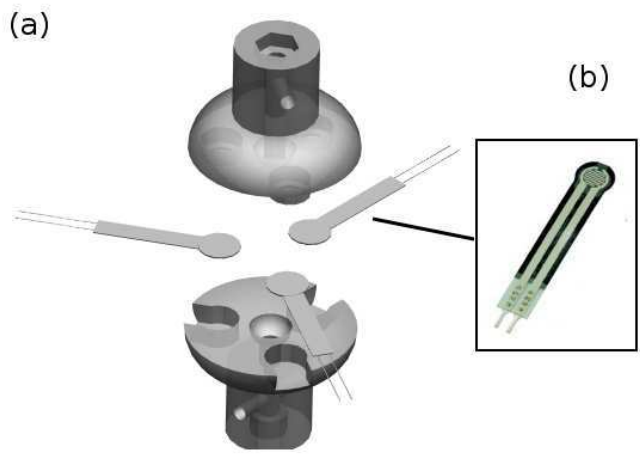

Fig. 2. Shown in (a) is an exploded CAD-view of the force sensing element that we used to deduce the normal and tangential forces at the contact point. It is based on a layer of force sensing resistors (b) that register the normal force and moments in the cut-surface of the sensor. A typical resistance vs. force characteristic of such a FSR is shown in (c) [12].

\section{Test setup}

For the terrain classification, artificial terrains with different geometrical shapes and surfaces textures were mounted onto the testbed (Fig. 3). In a first series, experiments were performed for four different terrain shapes: a convex and a concave cone, a convex hemispherical bulge, and a concave hemispherical indentation (Fig. 4a). The radius of the spheres was equal to the foot radius and the indention 
was made $20 \mathrm{~mm}$ deep. All samples were produced from rapid-prototyping material (Objet FullCure720) without any additional surface-treatment.

In a second series, flat terrain with different surface properties was investigated. To this end, we used a very low friction PTFE coating and three different types of abrasive paper with an ISO/FEPA grit designation of 60, 120, and 240 [13] (Fig. 4b).

The samples were mounted below the foot and the knee joint angle was defined to be zero when the shank tube was in vertical position. The symmetry axis of the samples was aligned with the axis of the shank tube. As the hip was not actuated, the contact force between foot and ground surface was created solely by the weight of the leg.

During the predefined leg motion, the analog signal of the FSR was low-pass filtered and amplified in an analog circuit, then sampled, and finally sent from a microprocessor (Microchip DSPIC33FJXMC710) via the UART interface to the main processing unit on a standard laptop computer (PC). Sensor data was sampled with a frequency of $50 \mathrm{~Hz}$.

PID position control on joint level was employed in the knee actuator by a Maxon EPOS 24/5 servo controller. The knee position profile generated by the PC was transmitted via CAN-bus to the motor controller, while the sensor values of the actuator, i.e. motor current, angular position, and angular rate, were sent back to the $\mathrm{PC}$, on which data pre-processing, feature extraction of the sample data and the classification algorithm were conducted.

(a)

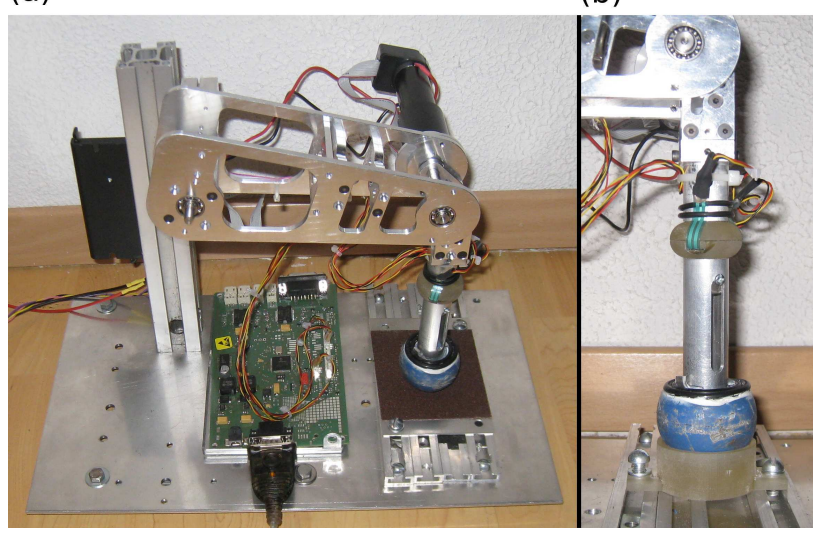

Fig. 3. To facilitated testing, one of the legs was separated from the main body and mounted to the sample holder of a testbed. Hip adduction/abduction was blocked, leaving only two degrees of freedom, of which only the knee joint was actuated. Different terrain samples could be fastened to this setup, which is shown as an overview in (a). (b) shows the detail of the shank with the incorporated force sensing element placed on a concave hemispherical indentation as terrain sample.

\section{Measurement cycle}

To obtain meaningful sensor data for a terrain shape and surface classification, the knee joint was actuated in two different manners: In the first set of experiments, the knee-joint oscillated slightly with an amplitude of about one degree, and a frequency of about $0.5 \mathrm{~Hz}$. Fig. 5 shows the measured knee (a)

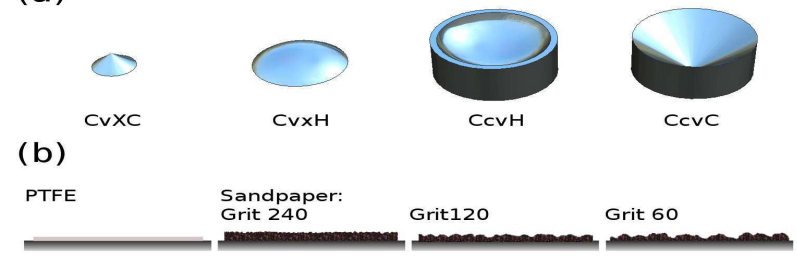

Fig. 4. Terrain in four different geometrical shapes (a) was mounted in the experimental testbed. As shape samples we used a convex cone ( $\mathrm{CvxC})$, a convex hemispherical bulge $(\mathrm{CvxH})$, a concave hemispherical indentation $(\mathrm{CcvH})$, and a concave conical bulge $(\mathrm{CcvC})$. They were produced by rapidprototyping without additional surface treatment. In a second series, a flat surface coated with Teflon and three different types of abrasive paper (b) was used to simulate standing on different coefficients of friction.

angle and the corresponding motor current when the leg is moving against a concave hemisphere. It also depicts the corresponding measurement of the three force sensors. This data was recorded and used for feature generation. Ideally, the signal amplitudes should be symmetrical about the neutral position of the leg oscillation, however, effects such as the non-linearity of sensors, backlash in the gears, or elasticity and hysteresis of the foot deteriorate the actual signals. The knee oscillation was used to determine the geometrical shape of the ground surface (shape classification).

(a)

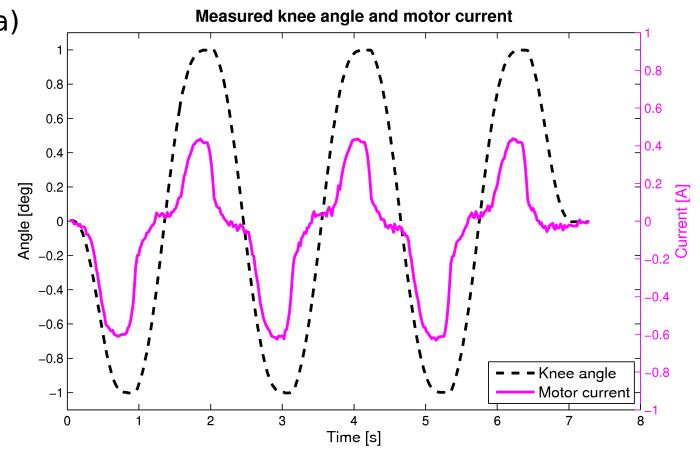

(b)

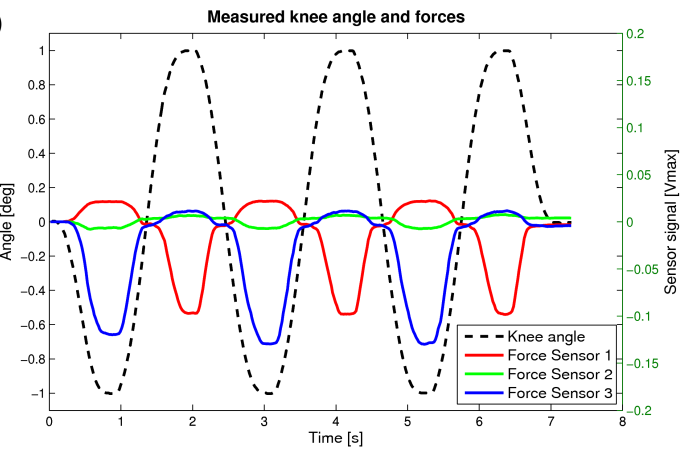

Fig. 5. Sample measurement of the knee angle, the corresponding motor current (a), and the three force sensor readings (b) while the leg is tested on a concave spherical indention.

For the surface classification, the foot was placed on a terrain sample and a planar leg motion was generated by 
rotating the knee by $10 \mathrm{deg}$. towards the leg mount, i.e. performing a scratching motion. Measuring the tangential ground contact force and the motor current during the motion allows the observation of static and dynamic friction effects. Fig. 6 shows an example plot of this motion on a PTFE coated surface. Provided are the motor current, as well as the time and frequency representation of the three force sensor readings.

(a)

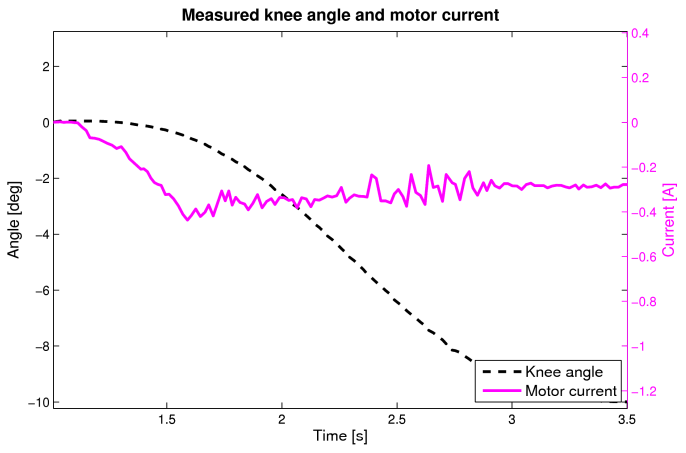

(b)

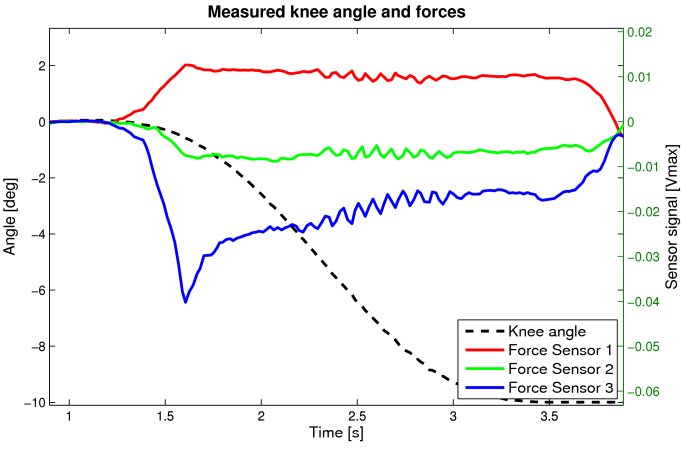

(c)

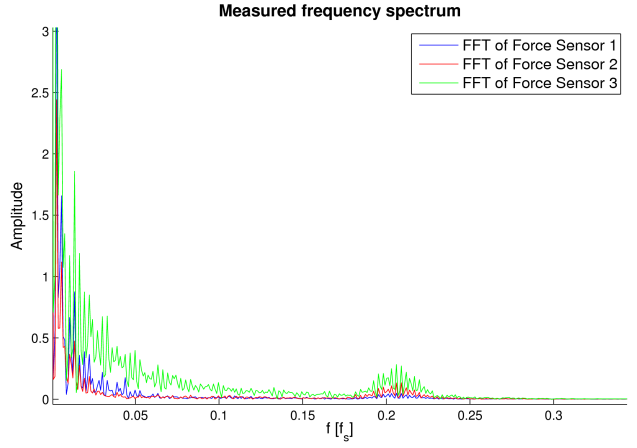

Fig. 6. Sample measurement of the knee angle, the motor current (a), the time traces of the force sensor signals (b), and the corresponding frequency domain representation (c, Frequency in $\%$ of the sample frequency $f_{s}, 50$ Hz.) on a PTFE-coated surface.

\section{E. Classification}

1) Overview: Fig. 7 shows a schematic of the classification process. After the execution of a measurement cycle, the recorded sensor data was preprocessed and features were extracted. This data was then used to either train the classifier or to evaluate its performance on a separate set of test data. The samples for terrain shape classification consisted of 25 training samples and 20 test samples for each of the four classes (180 samples total), and the classification of the terrain surface was based on 25 training samples and 15 test samples for every terrain surface (160 samples total).

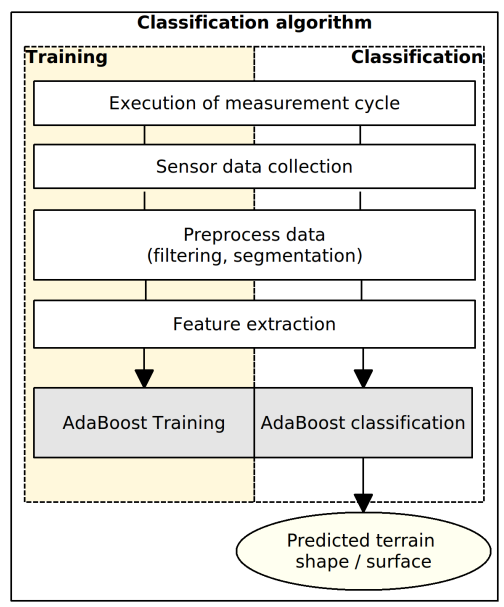

Fig. 7. Overview of the training/classification process for automated terrain characterization.

2) Classification method: We employed a multiclass variant of the binary AdaBoost classifier to differentiate the terrain surfaces and terrain shape samples. AdaBoost is a binary classification algorithm that combines a weighted ensemble of weak-classifiers into a strong classifier. The only requirement of each weak-classifier is that its accuracy is better than random guessing, that is better than 0.5 in a two-class case. The input to the algorithm is a set of labeled training data $\left(\mathbf{f}_{i}, l_{i}\right), i=1, \ldots, N$, where each $\mathbf{f}_{i}$ is an example and $l_{i}=\{+1,-1\}$ indicates whether $\mathbf{f}_{i}$ is positive or negative. In a series of rounds $t=1, \ldots, T$, the algorithm generates repeatedly a weak classifier $h_{t}\left(\mathbf{f}_{i}\right)$, in our case a decision stump, using a weight distribution $w(t)$ over the training examples. A decision stump defines a single axis-parallel partition of the space. The idea of the algorithm is to adapt the weight distribution $\mathbf{w}(t)$ at each round such that weak classifiers are trained on the mistakes of the previous one. The final strong classifier $H\left(\mathbf{f}_{i}\right)$ is a weighted majority vote of the $T$ best weak classifiers: $H\left(\mathbf{f}_{i}\right)=\operatorname{sign}\left(\sum_{t=1}^{T} \alpha_{t} h_{t}\left(\mathbf{f}_{i}\right)\right)$

Large weights are assigned to good weak classifiers whereas poor ones receive small weights. In our case we need to classify a sample of terrain surface (or shape) among multiple classes. We therefore face a multi-class classification problem for which we follow a one-vs-all strategy: when training a terrain surface class (or shape), all the features of the samples belonging to the considered class are treated as positive samples and all the features of the samples of the other classes are tagged as negative. This operation is computed for each class. When a unobserved sample feature $\mathbf{f}_{i}$ is presented for classification, it is processed by each learned classifier and the resulting class label is defined by the classifier with the larger likelihhod: 
$p\left(\mathbf{f}_{i} \mid \theta_{c}\right)=\frac{\sum_{t=1}^{T} \alpha_{t}^{c} h_{t}^{c}\left(\mathbf{f}_{i}\right)}{\sum_{t=1}^{T} \alpha_{t}^{c}}$, where $\theta_{c}$ represents the learned classifier for the class $c$.

3) Features: For every preprocessed sample vector $\mathbf{s}$ (consisting of three force sensor recordings and one current measurement), the following features have been computed and were used for classification.

1) The standard deviation $\sigma_{\mathbf{S}}$ of $\mathbf{s}$ in the time domain. The elements $s_{i} \in \mathbf{S}$ are considered, in this case, Gaussian distributed.

2) The maximum/minimum $\max =\max \left(s_{i}\right)$, min $=$ $\min \left(s_{i}\right), \in \forall i=1 \ldots n, s_{i} \in \mathbf{s}$

This ideally reflects the maximum force magnitude which occurs at the transition from static to dynamic friction of the leg.

3) The mean of $\mathbf{s}$ in the time domain. This can be interpreted as a metrics for the symmetry for the terrain shape measurement and reflects the dynamic friction during terrain surface measurements. The elements $s_{i} \in \mathbf{S}$ are considered, in this case, gaussian distributed.

4) Dominant frequency with $\mathrm{f}>5 \mathrm{~Hz}$ of force sensor and motor current sensor signals. The dominant frequency gives a hint about the vibration frequency that occurs in the dynamic friction phase (see Fig. $6 \mathrm{~b}$ and c).

5) The standard deviation $\sigma_{F S}$ of $S$ in the frequency domain.

In total, $4 \cdot 5=20$ features (five features for each of the four sets of sensor data) were collected per sample. The features are the same for the terrain shape and the terrain surface classification but may not have the same importance for both types (e.g. measuring the vibration frequency does not give valuable information for the terrain shape classification). However, the weighting and the selection of the individual features is managed by the training process of AdaBoost.

\section{RESUlts}

Table III shows the confusion matrix of the terrain shape classification to reflect the performance of the classificator. Shown in each row and column are the results for the convex cone $(\mathrm{CvxC})$, the concave cone $(\mathrm{CcvC})$, the convex hemispherical indentation $(\mathrm{CvxH})$, and the concave hemispherical indentation $(\mathrm{CcvH})$. The diagonal elements of this matrix represent the correctly classified samples while the other elements stand for wrongly predicted classes. The two parameters Success Rate (SR) and False Alarm Rate (FR) are used to evaluate the performance of the classification. The success rate is the ratio between the number of elements of a given class that have been classified successfully and the total number of elements in this class. The false alarm rate, on the other side, describes the number of elements wrongly classified as a certain class compared to the total number of elements that do not belong to this class.

In our experiments, very high success rates were achieved for the classification of the convex samples $(100 \%$ for the cone and $80.0 \%$ for the hemisphere). Concave shapes, on the other side could achieve success rates of $100 \%$ for the conical
TABLE III

CONFUSION MATRIX OF THE TERRAIN SHAPE CLASSIFICATION

\begin{tabular}{l|l|l|l|l|} 
& \multicolumn{5}{|c|}{ Terrain shape of sample } \\
Classified as & CcvC & CvxC & CcvH & CvxH \\
\hline CcvC & 20 & 0 & 1 & 0 \\
\hline CvxC & 0 & 20 & 0 & 4 \\
\hline CcvH & 0 & 0 & 19 & 0 \\
\hline CvxH & 0 & 0 & 0 & 16 \\
\hline \hline SR [\%] & 100.0 & 100.0 & 95.0 & 80.0 \\
\hline FR [\%] & 1.7 & 6.7 & 0.0 & 0.0 \\
\hline
\end{tabular}

TABLE IV

CONFUSION MATRIX OF THE TERRAIN SURFACE CLASSIFICATION

\begin{tabular}{l|l|l|l|l|} 
& \multicolumn{5}{|c}{ Terrain surface of sample } \\
Classified as & FT & G240 & G120 & G60 \\
\hline FT & 14 & 0 & 0 & 0 \\
\hline G240 & 1 & 12 & 7 & 5 \\
\hline G120 & 0 & 3 & 8 & 0 \\
\hline G60 & 0 & 0 & 0 & 10 \\
\hline \hline SR [\%] & 93.3 & 80.0 & 53.3 & 66.7 \\
\hline FR [\%] & 0.0 & 28.9 & 6.67 & 0.0 \\
\hline
\end{tabular}

shape and $95 \%$ for the hemisphere. This is a very good result for the prediction of small scale geometry, especially if we consider the fact that for the assessment of a selected foothold it is often sufficient to distinguish only between a convex and concave surface, as a concave surface shape can improve the grip and provide against slipping and sliding.

The algorithm performed slightly worse for the different terrain surfaces (Table IV). Within the different types of abrasive paper, an average of 2 out of 3 samples was classified correctly. As a positive result, the teflon coated surfaces (FT) have been predicted correctly with a probability of approx. 93.3\% and with no false alarms at all. Again, if we consider that to assess the quality of a foothold it might be sufficient to distinguish between low and high coefficients of friction, this is an overall satisfying result.

\section{Discussion}

\section{A. Conclusions}

In this paper we presented a novel approach to terrain shape and terrain surface classification for legged robots. A custom-made low cost force sensing device was integrated in a robotic leg to obtain haptic feedback that resulted from a prescribed knee joint actuation. A multiclass AdaBoost classifier has been trained with real-world data samples, which included features computed from motor current measurements as well as from contact force recordings. The classifier could reliably distinguish four different surface types and discriminate between concave and convex footholds. Even with the limited amount of available training data and with the cheap and noisy sensors that were used as base for the feature generation, the classifier showed a satisfying performance.

While these results were obtained in a simplified test setup, they pave the way for the application of terrain 
classification methods in actual legged robots and for the estimation of terrain properties in an unknown environment. This would be very helpful in the assessment of the quality of a selected foothold: Using haptics to evaluate the local surface properties before the weight of the robot is shifted onto the corresponding foot will help deciding whether a new and different placement of this foot is indicated, and hence improve the overall stability of locomotion in rough and highly unstructured terrain. Secondly, the obtained haptic information can be included in a geometric map of the environment where it can be combined with data from other sensors. This will allow the application of additional learning algorithms, with the goal to reliably predict the haptic terrain properties and thus expedite the selection of proper footholds, for example, by combining haptic and visual information as it is already common for wheeled systems [18].

A missing link in this process is the deduction from terrain properties as they were estimated in this work to the actual 'quality' of footholds, i.e., answering the question whether a specific foothold provides the robot with a secure stance or not. Within the presented framework, a very direct way of tackling this issues is to place the robot in an actual environment, and to manually 'show' it footholds that have been labeled 'good' and 'bad' by the user. The automated classifiers can then learn directly on these categories. This will make it unnecessary to model characteristics of an environment by hand, as done in previous projects [19].

We are currently considering a variety of improvements to increase the performance of the terrain classification. This refers less to the quality of the classification which is already more than satisfying, but rather to the process that is necessary to obtain the required data. Currently, samples with a length of up to 2 seconds are necessary to extract sufficient information for the classification process. Such long sample duration is a clear disadvantage if we strive to utilize the presented method in an actual walking robot. Improving the quality of the force sensing unit and increasing the sample frequency might allow to prescribe smaller and faster motions to the knee joint, and hence generate sufficient information in a considerably shorter time.

Furthermore, including the currently disabled hip joint in the analysis, will grant the opportunity to extend the analysis to three dimensions. As in our current setup the joints are not backdrivable, this will require some changes in the measurement routine: Most importantly, in contrast to the passive loading of the sample that was utilized in this study, we will need to rely on active loading of the foot. While maintaining a constant contact pressure will also have a beneficial impact on the quality of the extracted features, it requires a force-controlled robotic system, a technique that is indispensable anyway when we intend to apply the method to an actual multi-legged robot. Such a full-scale application is a truly challenging endeavor, as it not only substantially increases the number of degrees of freedom involved in loading a single leg, but also limits the range of tests that can be performed with a single foot. For example, performing trials that involve large motions or high contact forces might compromise the stability of the current stance and must hence be avoided at all costs.

However, based on the very promising results that we obtained in this simplified study, we believe that the application of haptic terrain classification in an actual multi-legged robot is a challenge well worth to tackle.

\section{ACKNowledgments}

The authors gratefully acknowledge Oliver Baur, Martin Latta, Andi Lauber, and Attila Yilmaz for their contribution in the design and construction of the ALoF robot.

\section{REFERENCES}

[1] R.B. McGhee and A.A. Frank, "On the stability properties of quadruped creeping gaits", in Mathematical Bioscience, Vol. 3, 1968, pp. 331-351

[2] D.E. Orin, "Interactive control of a six-legged vehicle with optimization of both stability and energy", Ph.D. Thesis, The Ohio State University, 1976

[3] M. Vukobratovic, A.A Frank and D. Juricic, "On the stability of biped locomotion, IEEE Transactions on Biomedical Engineering, Vol. 17, No. 1, 1970, pp. 25-36,

[4] E. Garcia, J. Estremera and P. Gonzalez-de-Santos, "A classification of stability margins for walking robots", in Robotica, Vol. 20, Issue 6,2002 , pp. $595-606$

[5] K. Byl, A. Shkolnik, S. Prentice, N. Roy, and R.Tedrake (2009). "Reliable Dynamic Motions for a Stiff Quadruped" in Experimental Robotics, pp. 319-328

[6] J. R. Rebula, P. D. Neuhaus, B. V. Bonnlander, M. J. Johnson, J. E. Pratt "A Controller for the LittleDog Quadruped Walking on Rough Terrain" in International Conference on Intelligent Robots and Systems, 2007

[7] A. Krebs, C. Pradalier and R. Siegwart, "Comparison of Boosting Based Terrain Classification Using Proprioceptive and Exteroceptive Data",in Experimental Robotics, Springer Tracts in Advanced Robotics, Volume 54, 2009, pp. 93-102

[8] C. Weiss, H. Fröhlich and Andreas Zell, "Vibration-based terrain classification using Support Vector Machines", in International Conference on Intelligent Robots and Systems, 2006

[9] C. Brooks and K. Iagnemma, "Vibration-based terrain classification for planetary rovers", in IEEE Transactions on Robotics, Vol.21, No.6, 2005, pp. 1185-1191

[10] L. Ojeda, J. Borenstein, G. Witus and R. Karlsen, "Terrain Characterization and Classification with a Mobile Robot", in Journal of Field Robotics, Vol. 23, No. 2, 2005, pp 103-122

[11] R. Manduchi, A. Castano, A. Talukder and L. Matthies, "Obstacle Detection and Terrain Classification for Autonomous Off-Road Navigation", in Autonomous Robots, Vol. 18, 2005, pp. 81-102

[12] Interlink Electronics, FSR Force Sensing Resistor Integration Guide and Evaluation Parts Catalog, Version 1.0

[13] (2009) Webpage of Federation of European Producers of Abrasives, http://www.fepa-abrasives.org

[14] Y. Freund and R.E. Schapire, "Experiment with a new boosting algorithm", in Machine Learning: proceeding of the 13rd International conference, 1996, pp. 148-156

[15] L Spinello and R Triebel and R Siegwart, "Multiclass Multimodal Detection and Tracking in Urban Environments", Proc. of The 7th International Conference on Field and Service Robotics (FSR), 2009

[16] Y. Freund, R.E. Schapire, "Discussion of the paper "Arcing Classifiers" by Leo Breiman", in The Annals of Statistics, Volume 26, 1998, pp 824-832

[17] Y. Freund. R.E. Schapire, "A Short Introduction to Boosting", in Journal of Japanese Society for Artificial Intelligence, Volume 14, 1999, pp. 771-780

[18] C.A. Brooks, K.D. Iagnemma, "Self-Supervised Classification for Planetary Rover Terrain Sensing", Aerospace Conference, IEEE 2007.

[19] M.Kalakrishnan, J. Buchli, P. Pastor, S. Schaal, "Learning Locomotion over Rough Terrain using Terrain Templates" in International Conference on Intelligent Robots and Systems 2009. 